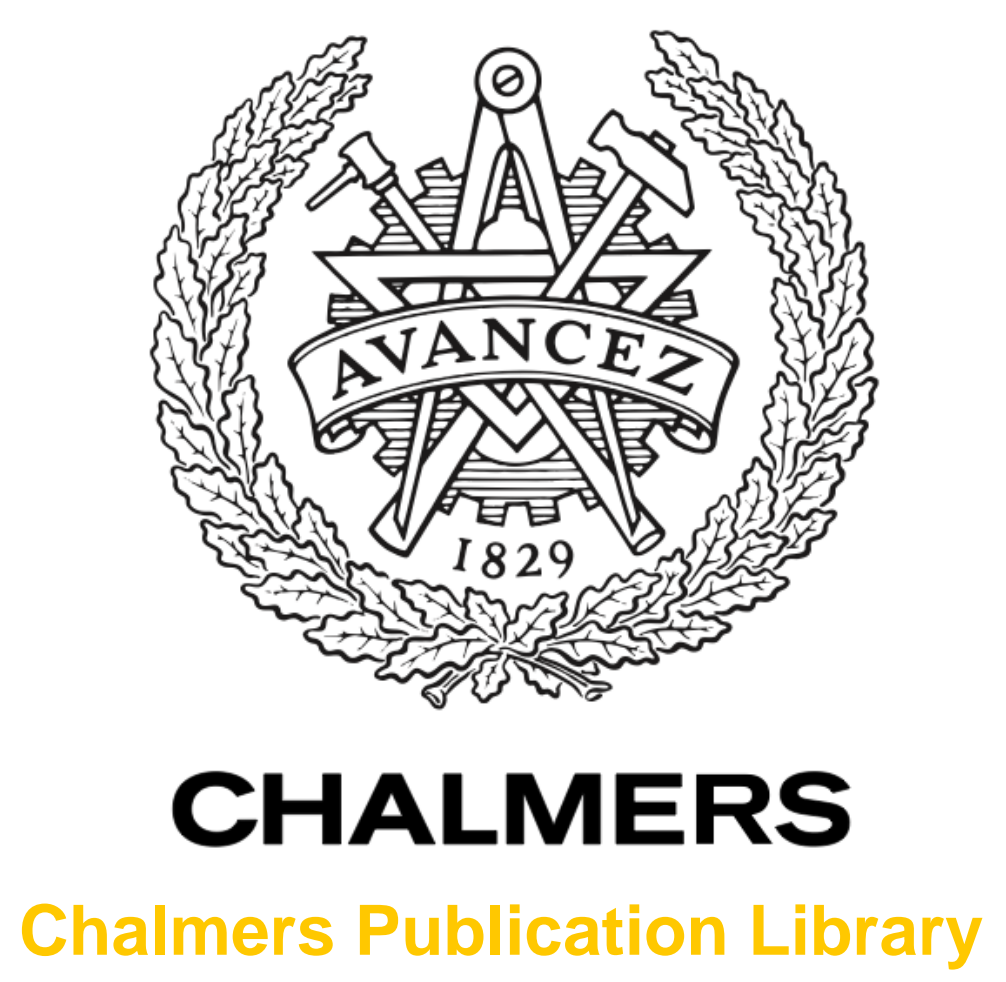

Magnetic Fields with the Atacama Large Millimeter/Submillimeter Array

This document has been downloaded from Chalmers Publication Library (CPL). It is the author's version of a work that was accepted for publication in:

Magnetic Fields in Diffuse Media, Astrophysics and Space Science Library, Volume 407

Citation for the published paper:

Vlemmings, W. (2015) "Magnetic Fields with the Atacama Large Millimeter/Submillimeter Array". Magnetic Fields in Diffuse Media, Astrophysics and Space Science Library, Volume 407 pp. 19-37.

http://dx.doi.org/10.1007/978-3-662-44625-6_2

Downloaded from: http://publications.lib.chalmers.se/publication/225706

Notice: Changes introduced as a result of publishing processes such as copy-editing and formatting may not be reflected in this document. For a definitive version of this work, please refer to the published source. Please note that access to the published version might require a subscription. 


\title{
Chapter 2 \\ Magnetic Fields with the Atacama Large Millimeter/Submillimeter Array
}

\author{
Wouter Vlemmings
}

\begin{abstract}
The Atacama Large Millimeter/submillimeter Array (ALMA) is one of the largest radio telescopes and is located at $5,000 \mathrm{~m}$ altitude in the Atacama desert in Chile. Its unprecedented sensitivity at extremely high angular and spectral resolution in the (sub-)millimetre wavelength regime, allows for countless advances in astrophysics. One of the areas in which ALMA can make unique contributions, is in that of the study of astrophysical magnetic fields. ALMA is expected to map the magnetic field geometry, and in some cases strength, in a large number of star forming regions, around evolved stars and planetary nebulae, and in nearby galaxies. This chapter provides examples of the amount of improvement ALMA offers the study of magnetic fields based on the current state-of-the-art and shortly introduces new tools that will be available to analyse (sub-)millimetre polarimetric observations.
\end{abstract}

\subsection{The Atacama Large Millimeter/Submillimeter Array}

The Atacama Large Millimeter/submillimeter Array (ALMA) is a large international (sub-)millimeter-wavelength radio telescope array in the Atacama Desert of northern Chile. It consists of a total of 66 antennas operating in the wavelength range from 0.4 to $4 \mathrm{~mm}$. Because of its unmatched sensitivity at very high spectral and spatial resolution, ALMA provides new insights into many astrophysical topics, from the formation of stars and planets to the evolution of the most distant galaxies. As ALMA is able to observe in full polarization mode, it can also reveal the magnetic field with a sensitivity and resolution that has not previously been possible.

ALMA is a partnership between Europe, North America (USA and Canada) and East Asia (Japan and Taiwan) in cooperation with the Republic of Chile. The ALMA project is coordinated by the Joint ALMA Office based in Santiago de Chile. The ALMA observatory is operated from the Operation Support Facility (OSF), which is situated at $\sim 2,900 \mathrm{~m}$ altitude. The array itself and the correlator are located at the

\footnotetext{
W. Vlemmings $(\bowtie)$

Chalmers University of Technology, Onsala Space Observatory, SE-439 92 Onsala, Sweden

e-mail: wouter.vlemmings@chalmers.se
} 


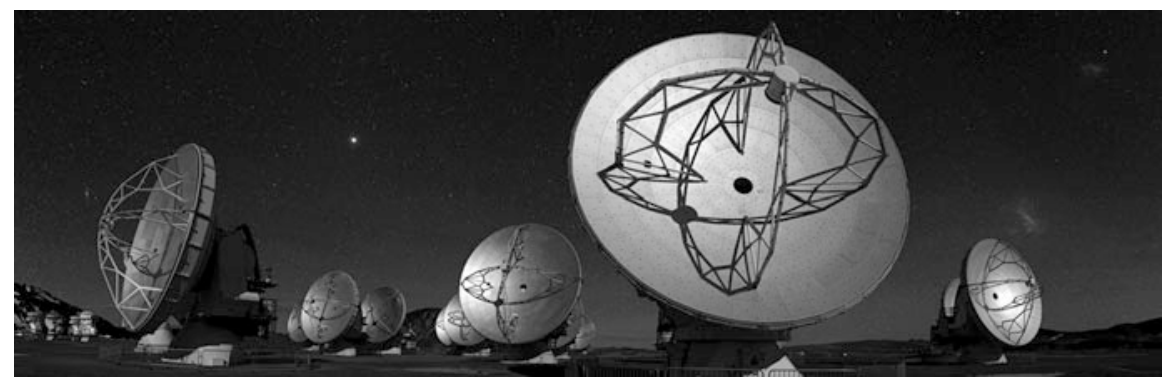

Fig. 2.1 Several ALMA antennas at the Chajnantor plateau. The 12-m diameter ALMA antennas are seen in the foreground, while in the far left a cluster of smaller 7-m antennas for the ACA are also visible. (Photograph credit: Babak Tafreshi)

Array Operation Site (AOS), at an altitude of 5,000 m (Fig. 2.1). ALMA is rapidly approaching its full operation, and has already observed a number of early science projects in a more limited configuration of $\sim 16$ to 40 antennas.

\subsubsection{ALMA Specifications}

The main ALMA array consists of 50 antennas with a diameter of $12-\mathrm{m}$. In addition, 12 antennas with 7-m diameter for interferometry and four 12-m diameter antennas for total power make up the Atacama Compact Array (ACA). This array can be used together with the main ALMA array to improve the imaging capabilities by providing short- and zero-spacing data.

The Atacama desert, at 5,000 m altitude, is one of the driest places on Earth. A typical value for the amount of water in the atmosphere, the precipitable water vapour (pwv), at the ALMA site is $\sim 1 \mathrm{~mm}$ (Fig. 2.2). As a result, the site provides excellent atmospheric transmission over the entire wavelength range covered by ALMA. The ALMA receiver bands and representative numbers are given in Table 2.1. In addition to those listed in the table, band $10(787-950 \mathrm{GHz})$ is planned shortly after completion of the array, and band 1 (around $40 \mathrm{GHz}$ ) and band 2 (around $80 \mathrm{GHz}$ ) might be added in the future. The sensitivity numbers reflect the dynamic scheduling nature of ALMA, with higher pwv allowed at the lower frequencies.

The main ALMA array can be configured with maximum baselines between $\sim 150 \mathrm{~m}$ to $\sim 16 \mathrm{~km}$. The ACA complements this by providing an array configuration with a diameter of $50 \mathrm{~m}$. At the shortest wavelength $(0.4 \mathrm{~mm})$ and largest configuration, ALMA will reach an angular resolution of $\sim 0.005^{\prime \prime}$, while thanks to the ACA it will still be possible to detect structure at the scale of $10^{\prime \prime}$. 


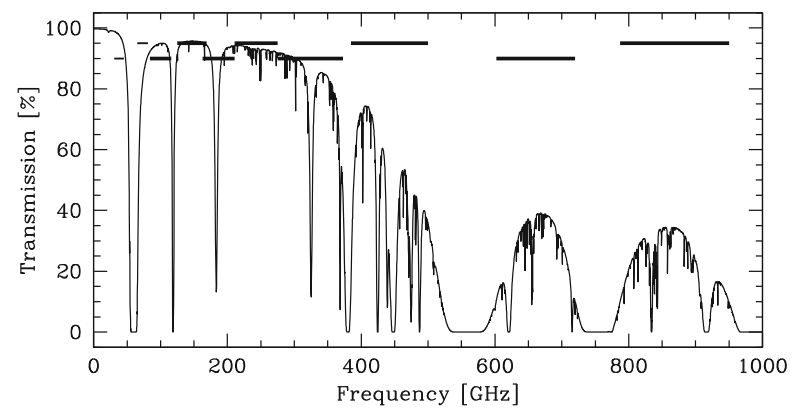

Fig. 2.2 The atmospheric transmission at the ALMA site. The curve shows the transmission for precipitable water vapour of $1.0 \mathrm{~mm}$. The horizontal bars from left to right indicate the ALMA frequency bands $1-10$. Bands 1 and 2 are potential upgrades

Table 2.1 The ALMA receiver bands

\begin{tabular}{l|l|l|l|l|l|l|l}
\hline & $\begin{array}{l}\text { Frequency } \\
\text { range } \\
\text { Band } \\
(\mathrm{GHz})\end{array}$ & $\begin{array}{l}\text { Wavelength } \\
\text { range } \\
(\mathrm{mm})\end{array}$ & $\begin{array}{l}\text { Angular resolution } \\
B_{\max }=150 \mathrm{~m}-16 \\
\left({ }^{\prime \prime}\right)\end{array}$ & $\begin{array}{l}\text { Line }^{\mathrm{a}} \\
\text { sensitivity } \\
(\mathrm{mJy})\end{array}$ & $\begin{array}{l}\text { Continuum } \\
\text { sensitivity } \\
(\mathrm{mJy})\end{array}$ & $\begin{array}{l}\text { Primary } \\
\text { beam } \\
\left({ }^{\prime \prime}\right)\end{array}$ & $\begin{array}{l}\text { Largest } \\
\text { scale } \\
\left({ }^{\prime \prime}\right)\end{array}$ \\
\hline 3 & $84-116$ & $2.6-3.6$ & $4.0-0.038$ & 8.9 & 0.05 & 61 & 37 \\
\hline 4 & $125-169$ & $1.8-2.4$ & $2.7-0.026$ & 9.1 & 0.06 & 51 & 32 \\
\hline $5^{\mathrm{c}}$ & $163-211$ & $1.4-1.8$ & $2.1-0.020$ & 1.3 & 0.06 & 33 & 23 \\
\hline 6 & $211-275$ & $1.1-1.4$ & $1.6-0.015$ & 13 & 0.10 & 25 & 18 \\
\hline 7 & $275-373$ & $0.8-1.1$ & $1.2-0.012$ & 21 & 0.20 & 19 & 12 \\
\hline 8 & $385-500$ & $0.6-0.8$ & $0.9-0.009$ & 63 & 0.40 & 14 & 9 \\
\hline 9 & $602-720$ & $0.4-0.5$ & $0.6-0.006$ & 80 & 0.64 & 9.7 & 6 \\
\hline
\end{tabular}

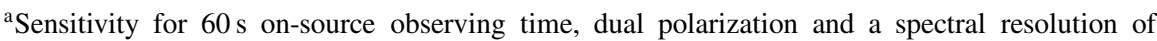
$1 \mathrm{~km} \mathrm{~s}^{-1}$

${ }^{\mathrm{b}}$ Sensitivity for using full continuum setup, with dual polarization, in $60 \mathrm{~s}$ on-source time ${ }^{\mathrm{c}}$ Initially only available on a limited number of antennas

The ALMA receivers have an instantaneous bandwidth of up to $8 \mathrm{GHz}$ in each of two polarizations that can be divided over four spectral windows of up to $2 \mathrm{GHz}$ each. The ALMA correlator is highly flexible and allows for a combination of multiple spectral modes simultaneously (Escoffier et al. 2007). In the case of full polarization correlation, it is possible to reach a spectral resolution from $976 \mathrm{kHz}$ $\left(1.28 \mathrm{~km} \mathrm{~s}^{-1}\right.$ at $\left.230 \mathrm{GHz}\right)$ down to $15 \mathrm{kHz}\left(0.02 \mathrm{~km} \mathrm{~s}^{-1}\right.$ at $\left.230 \mathrm{GHz}\right)$ in spectral line mode. The continuum observing mode produces 64 channels of $31.25 \mathrm{MHz}$ across the full $2 \mathrm{GHz}$ per spectral window.

At the time of writing, the full ALMA polarization capabilities have not yet been commissioned and only continuum observations are planned until 2015. Although depending on the performance in the individual bands, the goal is to reach at least a $0.1 \%$ accuracy, after calibration, in the linear polarization and a maximum absolute polarization angle accuracy of $6^{\circ}$. The reachable accuracy of the circular 
polarization is not yet determined, but it should, for compact sources, be of similar order.

\subsubsection{ALMA Science}

ALMA was originally designed with three specific science goals in mind. These goals are:

- The ability to detect spectral line emission from $\mathrm{CO}$ or CII in galaxies like the Milky Way at a red-shift of $z=3$, in less than $24 \mathrm{~h}$.

- The ability to probe the gas kinematics in protostars and protoplanetary disks around young Sun-like stars in molecular clouds out to $\sim 150 \mathrm{pc}$.

- The ability to provide high dynamic range images at an angular resolution of $0.1^{\prime \prime}$.

Considering its capabilities, ALMA will also make great strides in numerous other astrophysical topics, by, for example:

- imaging dust emission from galaxies out to $z=10$;

- studying the kinematics and physical conditions, including magnetic fields, in nearby galaxies through (polarimetric) imaging of the dust and molecular gas;

- observations of the gravitational collapse of molecular clouds as well as the formation of disks and jets around low-mass and high-mass protostars, including a study of the role of magnetic fields;

- imaging and model refinement of Solar System objects, such as planets, comets, asteroids and Kuiper Belt objects, as well as the Sun itself;

- making a detailed analysis of the dust formation, mass loss, shaping and magnetic fields in the circumstellar envelopes of evolved stars and planetary nebulae, as well as around supernovae.

Obviously, the sensitivity of ALMA together with its polarization capabilities provides a significant step beyond the currently available instruments in the ALMA wavelength range. In particular, ALMA will be able to deliver polarization data of scientific interest even when polarimetry is not the primary purpose of the observations. In Sect. 2.3, the potential of magnetic field studies with ALMA will be described compared to the current state-of-the-art in submillimetre and millimetre polarimetric observations.

\subsection{ALMA Polarization Calibration}

Much of the ALMA polarization science will be focused on linear polarization observations, although circular polarization measurements, in particular those of molecular Zeeman splitting, will also have several interesting applications. ALMA 
will be equipped with linearly polarized feeds, which is suboptimal for linear polarization observation (though for band seven quarter wave plates for obtaining dual circular polarization might be available). Assuming, following Cotton (1998), the linear approximation to the polarized antenna feed response and ignoring higher order terms in instrumental and source polarization, the $X$ and $Y$ linear feed crosscorrelations between antenna $n$ and $m$ are given by:

$$
\begin{aligned}
X X & =g_{n X} g_{m X}^{*}[I+Q \cos 2 \chi+U \sin 2 \chi] \\
X Y & =g_{n X} g_{m Y}^{*}\left[\left(d_{n X}-d_{m Y}^{*}\right) I-Q \cos 2 \chi+U \sin 2 \chi+i V\right] \\
Y X & =g_{n Y} g_{m X}^{*}\left[\left(d_{n Y}-d_{m X}^{*}\right) I-Q \cos 2 \chi+U \sin 2 \chi-i V\right] \\
Y Y & =g_{n Y} g_{m Y}^{*}[I-Q \cos 2 \chi-U \sin 2 \chi] .
\end{aligned}
$$

Here the individual antenna polarization gain terms are given by $g$, which are complex factors representing the effects of the atmosphere and uncorrected electronics. The leakage terms $d$ are also complex factors representing the fraction of the orthogonal polarization corrupting a given feed. The Stokes values are given by $I$ (total intensity), $Q$ and $U$ (linear polarization) and $V$ (circular polarization). Finally, $\chi$ is the parallactic angle, which corresponds to the rotation of the antenna feed seen from the source as it is being tracked during the observations.

While first order errors in the relative gains of the polarization feeds $X$ and $Y$ cancel out for calibration of the total intensity $I$, using a point source in the phase center, additional calibration of the $g$-terms is needed when interested in polarization. In particular, as seen in Eq. (2.1), any uncorrected phase difference between the two orthogonal polarized feeds, will couple the polarizations. This $X-Y$ phase difference $\left(\phi_{X Y}\right)$ cannot be determined by observing an unpolarized source. Uncorrected short term phase fluctuations between the feeds for the different telescopes in the array will further couple the total intensity and polarization and will introduce significant instrumental effects. Using linear feeds, the $X-Y$ phase difference needs to be known to much better than one degree to reach the goal of $0.1 \%$ polarization accuracy.

The exact calibration strategy would depend on how well the calibrator properties are known. One can identify three different possibilities:

1. Unpolarized calibrator: Only one observing scan is required for calibration of the $d$-terms. The antenna $d$-terms are calibrated and only one unknown complex number remains, which can be determined as part of the $g$-terms. The phase difference $\phi_{X Y}$ stays unknown.

2. Polarized calibrator with known polarization: A single scan will also be sufficient with only a remaining amplitude factor in $d_{X}$ and $d_{Y}$ that can be calibrated with the $g$-terms. The phase difference $\phi_{X Y}$ can be determined from the known polarization characteristics.

3. Polarized calibrator with unknown polarization: The calibrator needs to be observed at least three times over a range of $90^{\circ}$ or more in parallactic angle. 
The $d$-terms are calibrated with a single remaining complex number absorbed in $g$, but the phase difference $\phi_{X Y}$ remains unknown.

As is evident from these three cases, the phase difference $\phi_{X Y}$ can only be determined by observing a source with known polarization, while calibration of the $d$-terms needs either an unpolarized source or one with accurately known polarization. As such sources are rare in the ALMA wavelength range, it is thus likely, that in several cases, the calibration of the $d$-terms will require observations spanning a sufficient parallactic angle range, making polarization snapshot observations difficult. In any case, the stability in time and frequency of the $d$-terms and of $\phi_{X Y}$ will be critical but is yet undetermined for ALMA.

An additionally challenging aspect will be the need for an accurate polarization calibration across the entire primary beam, as many sources of interest for ALMA will have extended polarization. The instrumental gain and polarization leakage terms, as well as $\phi_{X Y}$ will depend on the location in the primary beam. Assuming the polarized beam is stable, a correction of these effects will require frequent observations by the ALMA project of a complete Stokes representation of the primary beam pattern.

Finally, the combination of the ACA and total power antennas could be needed for polarization mapping of extended sources. This requires in particular an observing scheme that properly calibrates the polarization of the total power antennas. As single dish calibration of polarization is time consuming and non-trivial, it is potentially possible to calibrate the total power antennas by including them into the full array during calibrator observations.

For the remainder of this chapter, it will be assumed that all calibration is performed optimally and the goal of $0.1 \%$ polarization accuracy is reached in both linear and circular polarization.

\subsection{Magnetic Field Observations with ALMA}

\subsection{1 (Sub-)Millimetre Polarization}

It is clear that magnetic fields are ubiquitous throughout the entire Universe and play an important role in a variety of astrophysical environments. A number of these environments, and the details on how polarized emission is generated under the influence of a magnetic field, will be described in other chapters of this book. As is evident from the science topics that its observations will address, ALMA will provide a breakthrough in observational diagnostics of the magnetic field in a number of astrophysical environments. Here, the improvement gained with ALMA in the observations of magnetic fields in Galactic star forming regions and molecular clouds, in nearby galaxies and around evolved stars will be addressed. But in addition, ALMA polarization will be able to provide important constraints on, for 
example, maser pumping and radiative transfer theory, galactic dust properties and models of $\operatorname{Sgr} \mathrm{A}^{\star}$.

\subsubsection{Linear Polarization}

At submillimetre wavelengths, linear polarization can be observed in both continuum dust emission as well as in the radiation originating from certain molecular line transitions. The dust continuum linear polarization, at far-IR and (sub-)millimetre wavelengths, arises from aligned and spinning dust grains. While the exact mechanism involved in the dust alignment is still uncertain (see e.g. Lazarian 2003, and further chapters in this book), all observations to date indicate that the alignment is with respect to the magnetic field. Only in the coldest and densest gas, as found in starless molecular clouds, are there indications that grains are only poorly aligned. The detectable fraction of linear polarization of dust emission is typically of the order of a few percent (e.g. Greaves et al. 1999). If at the same time, one has information on the turbulent velocity in the region probed by the polarization observations, the use of the Chandrasekhar-Fermi method (Chandrasekhar and Fermi 1953) can then provide a measure of the magnetic field strength in the plane of the sky using:

$$
B_{\mathrm{pos}}=\sqrt{4 \pi \rho} Q \frac{\delta v_{\mathrm{los}}}{\delta \phi} .
$$

Here $\rho$ is the average mass density, $\delta v_{\text {los }}$ is the line of sight velocity dispersion and $\delta \phi$ is the dispersion for the observed magnetic field vectors from the expected magnetic field structure after correcting for observational uncertainties. The dimensionless parameter $Q$ depends on the cloud structure. Turbulent cloud simulations indicate $Q \approx 0.5$ (Ostriker et al. 2001).

Linear polarization of molecular line emission can arise due to the GoldreichKylafis effect (Goldreich and Kylafis 1981, 1982). If even a relatively weak magnetic field is present, the molecular gas emission will be linearly polarized when the magnetic sublevels of the rotational states are exposed to anisotropic emission. Alternatively, polarized line emission can arise from molecules that have a preferred rotation axis as the result of strong infrared emission from a nearby source (Morris et al. 1985). Thus, in the presence of a strong IR source, the exact relation between the magnetic field and the linear polarization direction is not immediately clear. Additionally, while the linear polarization of dust emission is always perpendicular to the magnetic field direction, the molecular line linear polarization direction can be perpendicular or parallel to the magnetic field, depending on the direction of the velocity and radiation anisotropy (e.g., Cortes et al. 2005). However, with a careful analysis it is potentially possible to derive the magnetic field orientation from molecular line polarization measurements. The polarization fraction due to the Goldreich-Kylafis effect depends on several factors, such as the anisotropic 
radiation field, optical depth and angular momentum of the molecular transition involved, but is typically of the order of $1-10 \%$.

\subsubsection{Circular Polarization}

Circular polarization is generated in molecular line emission as a result of the Zeeman effect. When the emitting region is permeated by a magnetic field, the Zeeman effect causes a shift of the energy levels involved. This results in a splitting of the molecular line into three Zeeman components, one linearly polarized $\pi$ component and two oppositely circularly polarized $\sigma$ components. By measuring the frequency difference between the two circularly polarized components, it is possible to directly determine the magnetic field strength along the line of sight.

The Zeeman effect of different species scales with their magnetic moment. For paramagnetic molecules the magnetic moment is at the level of the Bohr magneton, while for non-paramagnetic molecules it scales with the nuclear magneton. This results in Zeeman splitting approximately three orders of magnitude smaller for non-paramagnetic molecules compared to paramagnetic molecules. For maser transitions from non-paramagnetic molecules, such as $\mathrm{H}_{2} \mathrm{O}$ and methanol, the Zeeman splitting has been measured (e.g., Fiebig and Guesten 1989; Vlemmings 2008). However, in the ALMA wavelength range, Zeeman splitting observations of high angular momentum transitions of non-paramagnetic molecules will be unlikely. Still, for several transitions, such as those from the paramagnetic molecules like CN (e.g., Falgarone et al. 2008) and SO, and potentially from atomic radiorecombination lines (e.g., Thum and Morris 1999), Zeeman splitting observations with ALMA will be possible.

\subsubsection{Star Formation and Molecular Clouds}

The specific importance of magnetic fields during star formation, both in the lowmass and high-mass regime, is still under debate, and will be covered in several of the other chapters. Polarization observations of star forming regions and molecular clouds have been able to probe the magnetic field at various scales and in a wide range of different density regimes. In the higher density regions (hydrogen number densities $n_{H_{2}}>10^{6} \mathrm{~cm}^{-3}$ ), close to the protostars, magnetic field measurements have mainly been made using maser observations in the centimeter wavelength regime. The linear and circular polarization maser observations have revealed large scale magnetic fields often aligned with outflows or torus/disc-like structures (e.g., Vlemmings et al. 2010; Surcis et al. 2009, 2012) and consistent with measurements of dust polarization (e.g. Fig. 2.3). The magnetic field strength ranges from a few $\mathrm{mG}$ in the less dense $\mathrm{OH}$ maser regions to several hundred $\mathrm{mG}$ in the shocked $\mathrm{H}_{2} \mathrm{O}$ maser regions (e.g., Bartkiewicz et al. 2005; Fish and Reid 2006; Sarma et al. 2001; Vlemmings et al. 2006). 


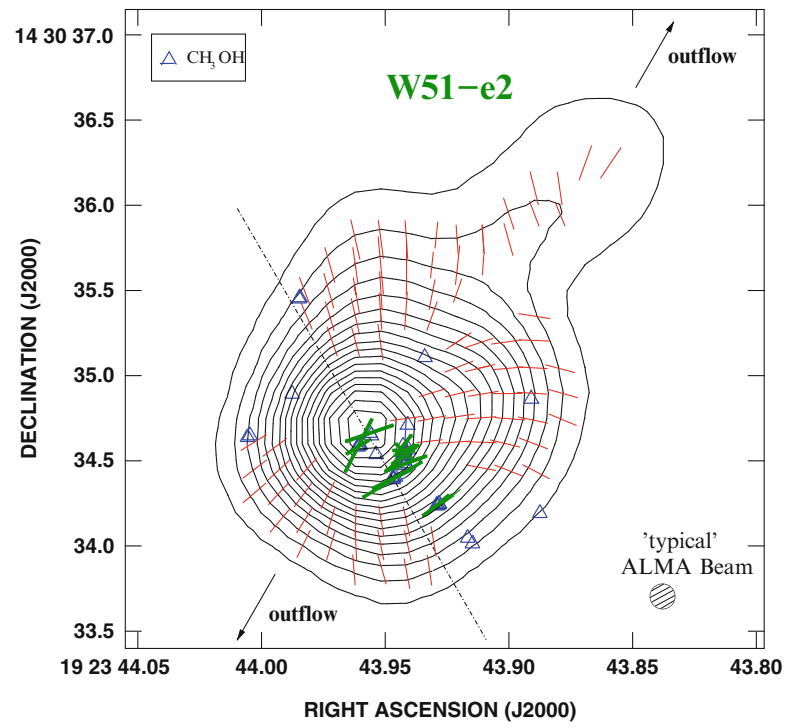

Fig. 2.3 The magnetic field map of core $\mathrm{e} 2$ of the massive star forming region W51. The contours are the $330 \mathrm{GHz}$ dust continuum (at 2, 4, 6, 8,10,15,20, ., $60 \times 60 \mathrm{mJy}^{\text {beam }}{ }^{-1}$ ) and the thin line segments are the $>3 \sigma$ magnetic field direction observed with the SMA (Tang et al. 2009). The SMA observations have a angular resolution of $0.7^{\prime \prime}$, corresponding to $\sim 4,000 \mathrm{AU}$. The triangles are the $6.7 \mathrm{GHz}$ methanol masers observed at $\sim 1$ mas $(\sim 5 \mathrm{AU})$ resolution with the European VLBI Network (EVN) (Surcis et al. 2012). The thick line segments are the magnetic field direction derived from the methanol maser polarization and agree perfectly with the dust observations. This indicates that the magnetic field maintains its regular structure across several orders of magnitude in scale

The magnetic fields in the lower density molecular clouds and protostellar envelopes have been observed using the Zeeman splitting of $\mathrm{HI}$ and non-masing $\mathrm{OH}$ transitions, as well as linear polarization from aligned dust grain (e.g., Crutcher et al. 1987, 1996; Girart et al. 2006). Additionally, several observations of CO polarization, attributed to the Goldreich-Kylafis effect, have been carried out (e.g., Girart et al. 1999; Beuther et al. 2010). It is particularly in these last two areas where important steps have been taken over the last few years, and where ALMA will provide significant further progress. A number of Submillimeter Array (SMA) observations have revealed the structure of the magnetic field around protostellar cores in both low-mass and high-mass star forming regions (e.g., Girart et al. 2006, 2009; Tang et al. 2009). These observations have revealed the hour-glass magnetic field morphology that is potential evidence for ambipolar diffusion as discussed in e.g. Mestel and Spitzer (1956). Taking as an example the observations of NGC 1333 IRAS 4A (Girart et al. 2006; Frau et al. 2011; Fig. 2.4) it is possible to estimate the improvements that ALMA will offer.

Example Polarization imaging of the $345 \mathrm{GHz}$ dust emission of NGC 1333 IRAS 4A, originally performed in Girart et al. (2006), was improved with further 


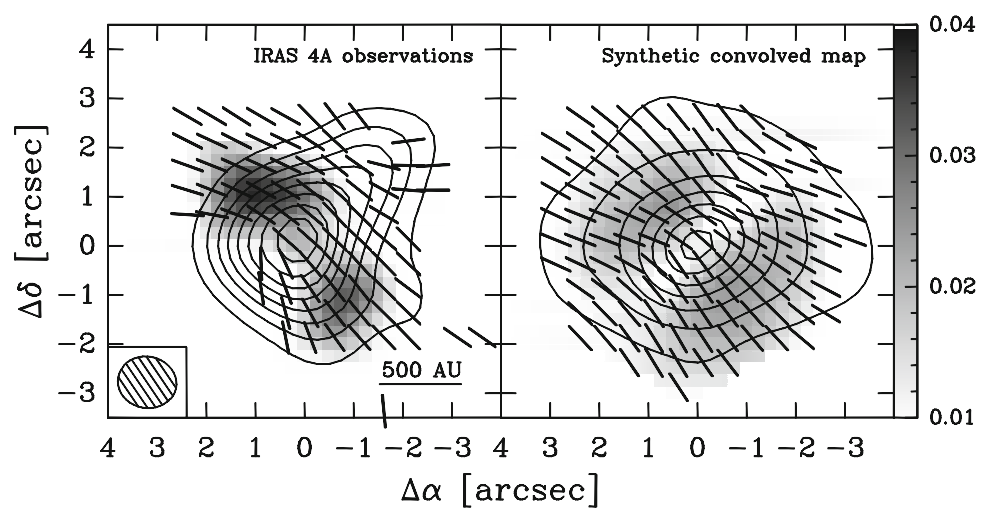

Fig. 2.4 Adapted from Padovani et al. (2012) and Frau et al. (2011). The left panel shows the dust polarization of NGC 1333 IRAS 4A using combined SMA sub-compact, compact and extended configuration observations. The contours indicate the $330 \mathrm{GHz}$ continuum emission in steps of $6 \sigma$, from 6 to $96 \sigma$, where $\sigma=20 \mathrm{mJy} \mathrm{beam}^{-1}$. The greyscale is the polarized emission (up to $38.3 \mathrm{mJy} \mathrm{beam}^{-1}$ and the line segments indicate the derived magnetic field direction. The panel on the right is a synthetic dust polarization map generated with the ARTIST module DustPol (Padovani et al. 2012; Sect. 2.4) produced with the same contour and intensity levels

observations in Frau et al. (2011). The resulting map, made from $8 \mathrm{~h}$ SMA tracks in the sub-compact, compact and extended configuration is shown in Fig. 2.4, left. The beam size was $1.24^{\prime \prime} \times 1.12^{\prime \prime}$ (corresponding to $434 \times 392 \mathrm{AU}$ at a distance of $\sim 350 \mathrm{pc}$ ). The polarized rms noise was $2.5 \mathrm{mJy}^{-1}$ beam $^{-1}$ and the peak total intensity and polarized flux were $1.03 \pm 0.02 \mathrm{Jy} \mathrm{beam}^{-1}$ and $38.2 \pm 2.5 \mathrm{mJy} \mathrm{beam}^{-1}$, respectively. To probe the full angular scale across which polarization is detected $\left(\sim 8^{\prime \prime}\right)$ at an angular resolution similar to that of the SMA, observations with ALMA at $345 \mathrm{GHz}$ will require the ACA. To reach the rms of $2.5 \mathrm{mJy}^{\text {beam }}{ }^{-1}$ per polarization, the main ALMA array would require less than $2 \mathrm{~s}$ on source integration time (the ACA would take approximately $3 \mathrm{~min}$, although because of the larger beam (6"), the required rms per beam will be less). Alternatively, in approximately $5.5 \mathrm{~h}$ on source integration time, ALMA would reach the limiting $0.1 \%$ linear polarization across the entire region where the SMA detected total intensity emission $\left(>20 \mathrm{mJy}\right.$ beam $\left.^{-1}\right)$.

Thus, ALMA would be able to produce polarization maps of similar quality to that of NGC 1333 IRAS 4A for $\sim 10$ sources in one observing track at a significantly improved angular resolution of $\sim 0.2^{\prime \prime}$ (assuming the settings are such that the polarization calibrator can be shared and observed over sufficient parallactic angle). Consequently, it could also detect the polarization for star forming cores at much larger distances, such as many of the massive star forming regions, or of much smaller mass. In one observing track it could image NGC 1333 IRAS 4A out to beyond $3 \mathrm{kpc}$ or with an order of magnitude smaller dust envelope mass. At the same time, with a spectral window placed on the $\mathrm{CO}(3-2)$ line, $0.1 \%$ linear polarization can then be detected in $1 \mathrm{~km} \mathrm{~s}^{-1}$ channels for a line strength of $\sim 1 \mathrm{Jy} \mathrm{beam}^{-1}$. 


\subsubsection{Evolved Stars and Planetary Nebulae}

At the end of their evolution, a majority of stars go through a period of high mass loss that is an important source for replenishing interstellar space with processed materials. During the asymptotic giant branch (AGB) phase, this mass loss produces circumstellar envelopes (CSEs), which are found to undergo a major modification during the rapid transition from AGB star to Planetary Nebula (PN). The standard assumption is that the initial slow AGB mass loss in a short time changes into a fast superwind generating shocks and accelerating the surrounding envelope (Kwok et al. 1978). However, a large fraction of PNe have asymmetric shapes, with the majority of the young PNe being bipolar. Thus, at some point during the evolution to a PNe the AGB stars must undergo a process in which the spherically symmetric outflow is altered to produce aspherical PNe morphologies. It has been shown that the energy and momentum contained in the outflows of young bipolar PNe is often orders of magnitude larger than can be provided by radiation pressure (Bujarrabal et al. 2001). The source of this energy has been argued to be magnetic fields, binary or disk interaction or a combination of these (see e.g. Balick and Frank 2002; Frank et al. 2007, and references therein).

As in star forming regions, the main source of magnetic field information around evolved stars has come from maser polarization observations. Ordered magnetic fields with a strength of the order a $\mathrm{mG}$ have been detected in the $\mathrm{OH}$ maser regions at large distances from the star e.g. Szymczak et al. (1998). Closer in, $\mathrm{H}_{2} \mathrm{O}$ masers also indicate the presence of a dynamically important magnetic field, with typical values of a few hundred $\mathrm{mG}$ e.g. Vlemmings et al. (2005). Finally, 43 and $86 \mathrm{GHz}$ $\mathrm{SiO}$ maser observations reveal field strengths of several Gauss at a distance of only a few stellar radii from the star, assuming a standard Zeeman interpretation (Kemball and Diamond 1997; Herpin et al. 2006). The current measurements are summarized in Fig. 2.5.

Although the exact relation between the magnetic field strength and the distance to the central star remains uncertain, it is likely that the measured fields dynamically influence the shaping of the outflow and, consequently, help shape asymmetric PNe. The magnetic field could also contribute to the stellar mass-loss mechanism (Falceta-Gonçalves et al. 2006). But crucial information on the magnetic field morphology is still lacking. Polarization observations of the masers in the envelope of the supergiant star VX Sgr indicates a large scale magnetic field with its configuration remaining similar from near the star out to several thousands of AU (Vlemmings et al. 2011). However, typically, maser observations only probe a limited number of lines-of-sights through the circumstellar envelope. Observations of thermal molecular line linear polarization, with the different species and transitions tracing different areas of the circumstellar envelope, will thus provide important insight in the relation between the magnetic field and the morphology of the circumstellar environment. 


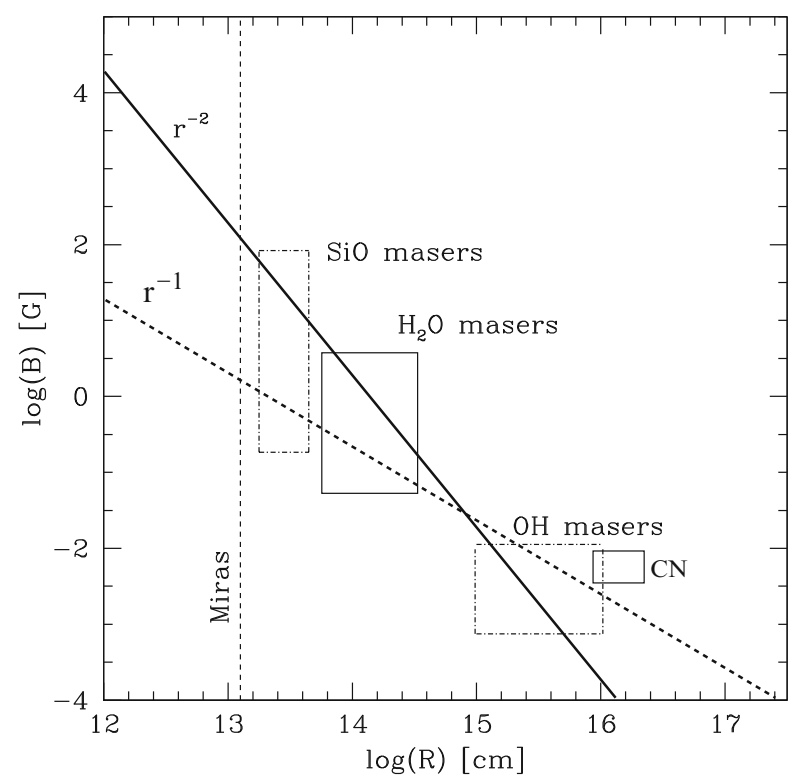

Fig. 2.5 Adapted from Vlemmings et al. (2005). The magnetic field strength vs. radius relation as indicated by current Zeeman splitting observations of the circumstellar environment of evolved stars. The boxes show the range of observed magnetic field strengths derived from the observations of $\mathrm{SiO}$ (e.g., Herpin et al. 2006; Amiri et al. 2012), $\mathrm{H}_{2} \mathrm{O}$ (e.g., Vlemmings et al. 2005) and $\mathrm{OH}$ masers (e.g., Wolak et al. 2012) as well as those from thermal $\mathrm{CN}$ circular polarization (Herpin et al. 2009). The thick solid and dashed lines indicate an $R^{-2}$ solar-type and $R^{-1}$ toroidal magnetic field configuration. The vertical dashed line indicates the stellar surface of a typical AGB star. Thermal molecular line polarization observations with ALMA will probe a wide range of distances (out to several times $10^{17} \mathrm{~cm}$ ) and can provide not only the morphology at those scales but also a plane-of-the-sky magnetic field strength using the Chandrasekhar-Fermi method

A tentative detection of linear polarization of $\mathrm{CS}(2-1)$ in the envelope of the AGB carbon star IRC+10216 was made using single dish observations (Glenn et al. 1997). Later, the first significant detection of linear polarization of $\mathrm{CO}(2-1)$ and $\mathrm{SiO}(5-4)$ was made in the envelope of IK Tau with the SMA (Vlemmings et al. 2012; Fig. 2.6). This was followed shortly afterwards with the detection of $\mathrm{CO}(3-2)$, SiS(19-18) and CS(7-6) linear polarization, again with the SMA, in the envelope of IRC +10216 (Girart et al. 2012). Detailed analysis of radiation and velocity anisotropies, together with multi-level polarization radiative transfer, are needed in order to properly evaluate the relation between the linear polarization and the magnetic field. If the observed polarization is due to the regular GoldreichKylafis effect, the observations confirm the presence of a large scale circumstellar magnetic field. In the case of IK Tau this field then appears to be related to the slightly elongated CO envelope morphology (Castro-Carrizo et al. 2010).

Beyond the AGB phase, magnetic field information is even more scarce. A small number of post-AGB and pre-PNe have had their magnetic fields measured, the 

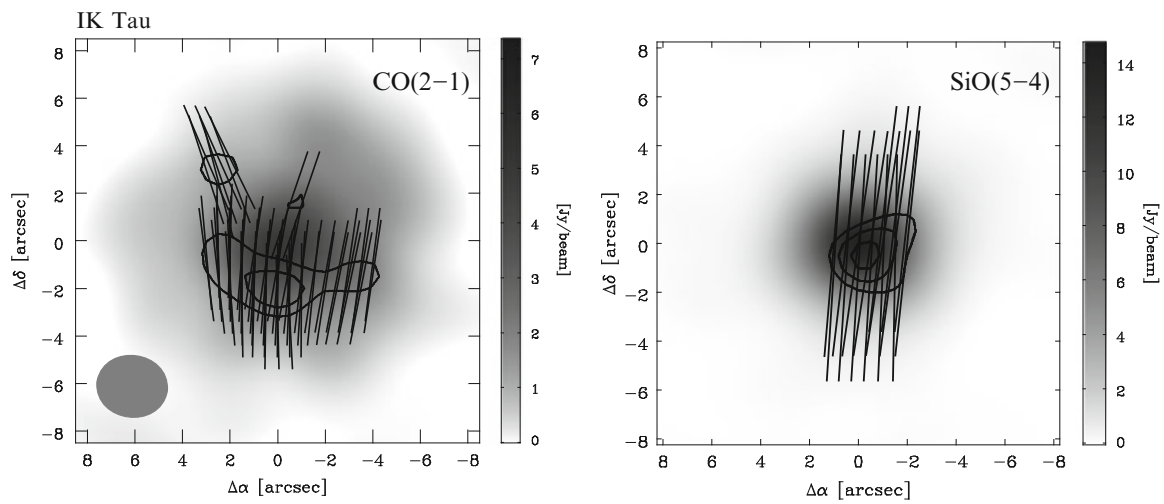

Fig. 2.6 From Vlemmings et al. (2012). The thermal molecular line linear polarization of $\mathrm{CO}(2-1)$ at $230 \mathrm{GHz}$ (left) and the $\mathrm{SiO}(5-4)$ at $217 \mathrm{GHz}$ in the circumstellar envelope of the AGB star IK Tau. The observations were done with the SMA in compact configuration. The contours indicate the 3,4 and $5 \sigma$ confidence levels for the polarized emission and the line segments show the linear polarization direction. While the $\mathrm{SiO}$ emission originates from closer to the star than the $\mathrm{CO}$ emission, the polarization direction is the same. This potentially indicates a preferred field direction throughout the stellar envelope. Due to the $90^{\circ}$ ambiguity in the magnetic field direction derived from the polarization vectors, the exact magnetic field orientation is not yet known. However, the preferred direction, either North-South or East-West, could be related to the observed East-West extent in the circumstellar envelope

most notable being the detection of a magnetically collimated jet from the "waterfountain' pre-PN W43A (Vlemmings et al. 2006). No convincing detections of magnetic fields on PN central stars have yet been made (Leone et al. 2011) and only very few display maser emission that can be used for polarization observations. Thus far, only the young PNe K3-35 has been confirmed to harbour a mG magnetic field in the circumstellar nebula (Gómez et al. 2009). The most convincing evidence for a large scale magnetic field in PNe comes from dust polarization mapping (Sabin et al. 2007).

Depending on the molecule and transition, the observed molecular line linear polarization fractions are between a few and $\sim 10 \%$. Those of the dust are of order of a few percent. The SMA can currently only detect such levels for the handful of evolved stars and planetary nebulae with sufficiently bright dust and/or molecular line emission. However, based on the SMA observations of IK Tau, it is clear that ALMA will provide the sensitivity to significantly expand this field of research. In addition, ALMA will allow for measurements of $\mathrm{CN}$ circular polarization (e.g. Herpin et al. 2009) in the envelopes of carbon rich AGB stars that host few masers.

Example The linear polarization of $\mathrm{CO}(2-1)$ in the envelope of IK Tau was observed with the SMA in a single $9 \mathrm{~h}$ track (Vlemmings et al. 2012; Fig 2.6). Despite observing in the compact configuration, a significant amount of flux was lost due to the extent of the CSE. The rms per $5 \mathrm{~km} \mathrm{~s}^{-1}$ channel in the polarization and total intensity images was $35 \mathrm{mJy}^{\text {beam }^{-1}}$ and the $\mathrm{CO}(2-1)$ peak emission was 
$7.5 \mathrm{Jy} \mathrm{beam}^{-1}$ in a $3.0^{\prime \prime} \times 2.6^{\prime \prime}$ beam. As for the dust polarization measurements in star forming regions, ALMA would be able to reproduce these observations in only a few seconds of observing time. Alternatively, it will produce an improved $(>10 \sigma)$ map with higher spatial resolution $\left(1^{\prime \prime} \times 1^{\prime \prime}\right)$ and better velocity resolution $\left(2 \mathrm{~km} \mathrm{~s}^{-1}\right)$ within a total observing time of $30 \mathrm{~min}$. Although IK Tau is one of the strongest $\mathrm{CO}$ emitting AGB stars, the use of the ACA would allow ALMA to recover more flux and thus observe the thermal molecular line polarization of about a dozen evolved stars out to $\sim 0.5 \mathrm{kpc}$ in a single observing track. Similarly, it will be possible to map individual objects with a $\mathrm{CO}(2-1)$ line strength of only $\sim 250 \mathrm{mJy} \mathrm{beam}^{-1}$ to a linear polarization of approximately $1 \%$ in a single $8 \mathrm{~h}$ observing run. Dust polarization at $230 \mathrm{GHz}$ will simultaneously be detectable at the $0.1 \%$ level for sources with a continuum flux of only $\sim 16 \mathrm{mJy} \mathrm{beam}^{-1}$. This would easily allow for dust polarization mapping of, for example, extended PNe.

\subsubsection{Nearby Galaxies}

In addition to Galactic magnetic field observations, ALMA will also provide new means of studying the magnetic field in nearby galaxies. Currently, the knowledge of magnetic fields in nearby galaxies mainly comes from observing the polarization of radio synchrotron emission e.g. Beck et al. (2005) and Faraday rotation analysis of local emission as well as that of background sources (e.g., Krause and Beck 1998; Gaensler et al. 2005). These observations and methods, as well as their interpretation, will be covered in other chapters.

The synchrotron polarization typically traces magnetic fields in the diffuse and warm interstellar medium and indicates a field strength ranging from $\sim 5 \mu \mathrm{G}$ in radio-faint galaxies to $\sim 100 \mu \mathrm{G}$ in starburst galaxies. Such field strengths indicate that magnetic fields are dynamically important. At higher densities, magnetic field probes are more limited. Field strengths up to $\sim 18 \mathrm{mG}$ were detected using $\mathrm{OH}$ megamaser emission in a number of starburst galaxies (Robishaw et al. 2008). The megamaser observations probe the magnetic field in dense regions, although it is not clear if these are the generally compressed interstellar medium of the nuclear starburst regions or even more dense molecular clouds.

With ALMA, it will now be possible to probe the magnetic field in the molecular clouds of the nearest galaxies by observing the polarized dust and molecular line emission. Previously, dust polarization of a few percent was observed in the central region of M82 (Greaves et al. 2000), but only very few polarization vectors at $15^{\prime \prime}$ resolution were mapped. More recently, the $\mathrm{CO}$ polarization, attributed to the Goldreich-Kylafis effect, was measured for six giant molecular clouds (GMCs) in M33 (Li and Henning 2011). From these SMA observations it was concluded that there exists a strong relation between the large scale galactic magnetic field and the field found in GMCs. With ALMA similar studies will be possible at higher angular resolution and of much more of the $\mathrm{CO}$ emitting molecular gas. 
Example The observations of the $\mathrm{CO}(2-1)$ polarization of $\mathrm{M} 33$ were also performed with the SMA (Li and Henning 2011). Although detailed information about the SMA observations is not available, it is clear from similar arguments presented earlier that ALMA would be able to reproduce the SMA results within a fraction of the time, even considering that the observational setup would require (pointed) mosaics. As an example project, we take the $\mathrm{CO}$ observations of the galaxy merger NGC 4194-also known as the Medusa galaxy (Aalto and Hüttemeister 2000), although this particular source is not observable by ALMA. Figure 2.7 shows the $\mathrm{CO}(1-0)$ distribution, tracing prominent dust lanes of the merger. The $\mathrm{CO}$ is extended over $\sim 25^{\prime \prime}$, which means that ALMA would be able to map the $\operatorname{CO}(1-$ 0 ) in a single pointing, but would need a small mosaic for the higher transitions (where also the ACA is needed to recover the extended emission). Using a single observing track with ALMA, the channel rms in $20 \mathrm{~km} \mathrm{~s}^{-1}$ channels at $\mathrm{CO}(1-0)$ is approximately $0.2 \mathrm{mJy}$ beam $^{-1}$. It would thus be possible to reach the maximum $0.1 \%$ linear polarization accuracy for the inner $\sim 10^{\prime \prime}$ of $\mathrm{CO}(1-0)$ emission shown in the right panel of Fig. 2.7. As the flux in the $\mathrm{CO}(2-1)$ transition is $2-3$ times larger, and the rms noise in a single track would be similar to that at $\mathrm{CO}(1-0)$ in the

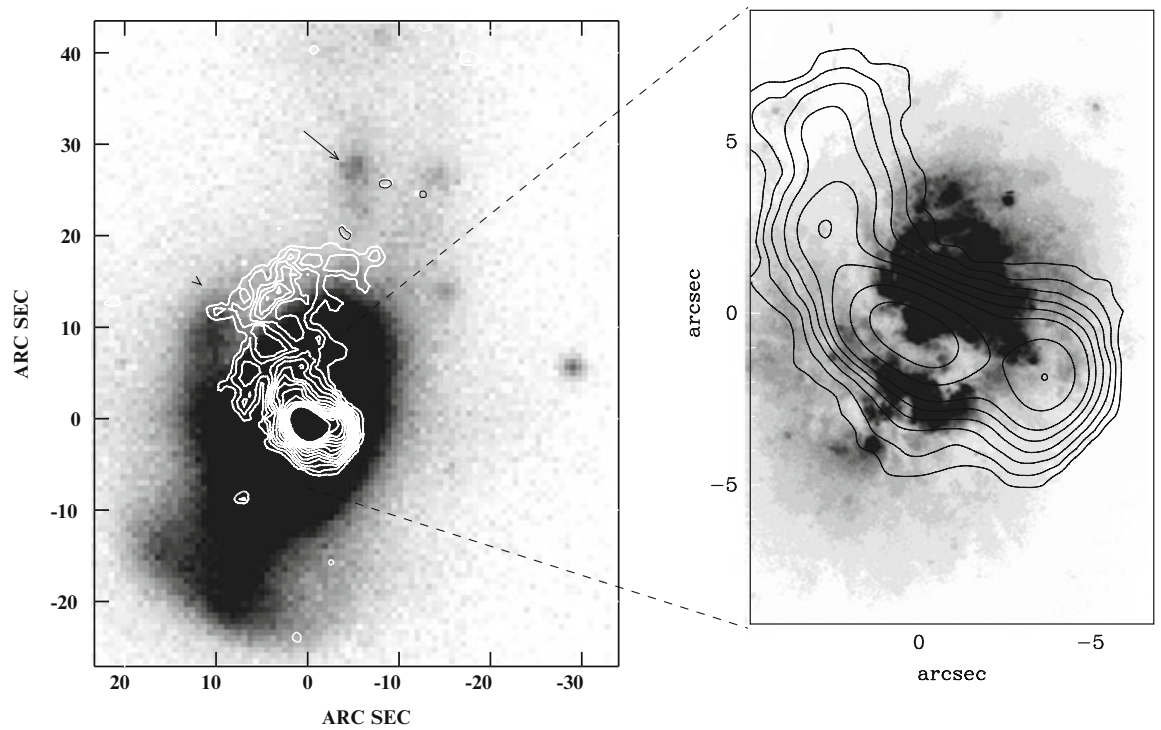

Fig. 2.7 Observations of the $\mathrm{CO}(1-0)$ transition at $115 \mathrm{GHz}$ of the "Medusa" merger galaxy NGC 4194 at $D=39 \mathrm{Mpc}$ (Aalto and Hüttemeister 2000). The observations were performed using four tracks at the Caltech six-element Owens Valley Radio Observatory (OVRO), providing a beam size of $2.5^{\prime \prime} \times 2.0^{\prime \prime}$ and an rms of $11 \mathrm{mJy}^{-1}$ beam $^{-1}$ in $20 \mathrm{~km} \mathrm{~s}^{-1}$ channels. The image to the left has the integrated $\mathrm{CO}$ contours overlayed on an optical R-band image. The image on the right displays only the emission from 2,506 to $2,570 \mathrm{~km} \mathrm{~s}^{-1}$ on an HST WFPC2 image. Contour levels are $0.54,2.6,2.7,3.8, \ldots, 31 \mathrm{Jy}_{\text {beam }}^{-1} \mathrm{~km} \mathrm{~s}^{-1}$. The emission extends over a total scale of $25^{\prime \prime}$. While this source is in the Northern hemisphere, ALMA will be able to detect the CO polarization for similar sources 
case of a small mosaic, polarization would be detectable for most of the emission. ALMA will thus be able to map the magnetic field for several nearby galaxies such as NGC 4194.

\subsection{ALMA Polarization Analysis Tools: The ARTIST Project}

The ARTIST (Adaptable Radiative Transfer Innovations for Submillimeter Telescopes) project ${ }^{1}$ was designed as a general model suite for comprehensive multidimensional radiative transfer calculations of dust and molecular line emission (Padovani et al. 2011). In addition to the model suite, which is based on the full three-dimensional radiative transfer code LIME (Line Modeling Engine, Brinch and Hogerheijde 2010), the ARTIST project will also provide the tools for modeling polarization at (sub-)millimetre wavelength. As the ARTIST uses a Python-based user interface and produces maps in the FITS format, it can straightforwardly be combined with the Common Astronomy Software Applications package (CASA) that is used for ALMA. In this way it will be possible to directly model the interferometric ALMA response to the polarized emission from model sources produced by the user.

The polarization analysis tools of ARTIST have two components, one for the continuum dust polarization and one for the thermal molecular line polarization. The dust polarization module (DustPol) is described in detail in Padovani et al. (2012). As it is based on LIME, DustPol can be combined with analytical as well as pregridded models and output from, e.g., magneto-hydrodynamical (MHD) models. An example of the package capabilities is shown in Fig. 2.8. Here, input from the MHD adaptive mesh refinement code RAMSES (Teyssier 2002; Fromang et al. 2006) is used to produce predictions for polarization observations of a rotating and collapsing core with different mass-to-magnetic flux. The power of DustPol can also be seen in Fig. 2.4, in which the SMA dust polarization observations of NGC 1333 IRAS 4A are compared with a synthetic dust polarization map made with DustPol. The model, with parameters from Frau et al. (2011), corresponds to a collapsing magnetized isothermal toroid as described by Allen et al. (2003).

The second component is the full polarization radiative transfer treatment of thermal molecular lines. The ARTIST line polarization module (LinePol) is mainly aimed at modeling the Goldreich-Kylafis effect on the emission of molecules such as $\mathrm{CO}$ (Kuiper et al. in prep.). It thus does not include radiative alignment effects. However, as it uses the multi-level and full three-dimensional radiative transfer capabilities of LIME, LinePol will predict the polarization response of molecular lines in a highly anisotropic radiation field.

\footnotetext{
${ }^{1} \mathrm{http} / / /$ youngstars.nbi.dk/artist
} 


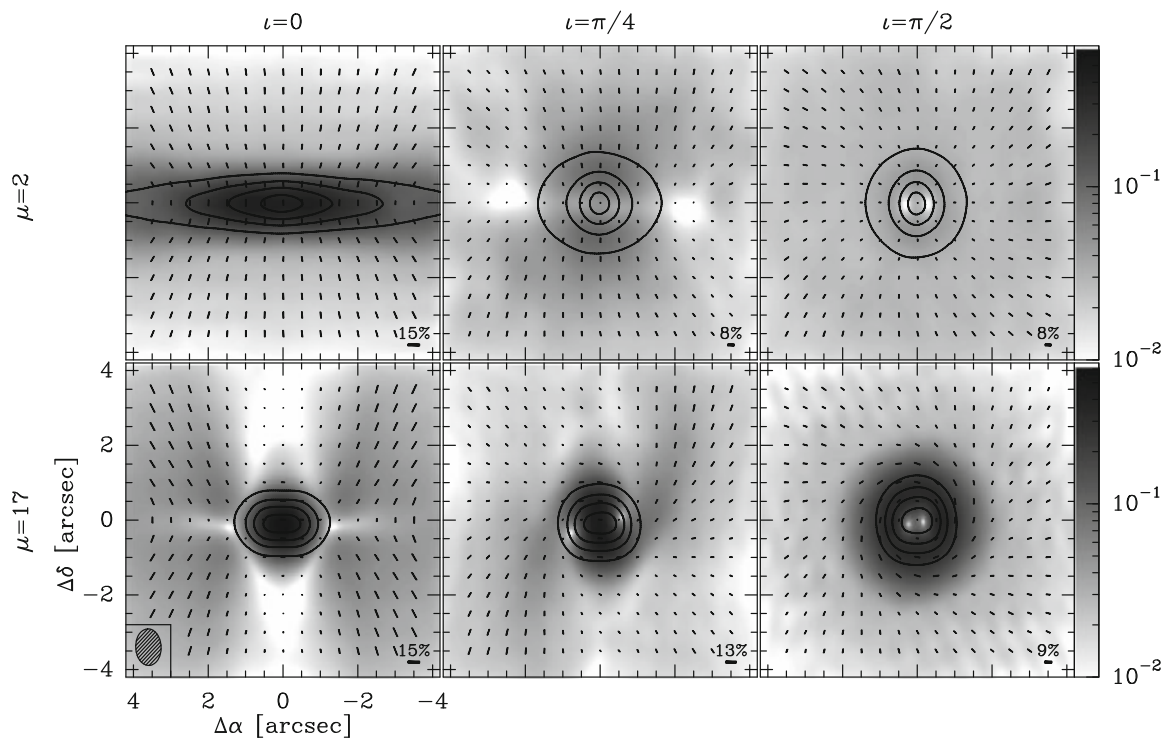

Fig. 2.8 Polarization maps for a collapsing and rotating magnetized core produced with the ARTIST dust polarization module DustPol (Padovani et al. 2012). The models were obtained using the RAMSES code. The top row shows the case of a strong magnetic field (mass-to-flux ratio $\mu=2)$ and the bottom row that for a weak magnetic field $(\mu=17)$. From left to right the inclination varies from edge-on to face-on. The source was assumed to be at $D=140 \mathrm{pc}$ with a dust temperature $T_{\text {dust }}=10 \mathrm{~K}$. The simulated ALMA beam, at $350 \mathrm{GHz}$, is $0.98^{\prime \prime} \times 0.68^{\prime \prime}$. The simulations show the core at an age of $t=2.4 \times 10^{4} \mathrm{y}$ with the magnetic field parallel to the rotation axis at $t=0$. Contours show 30,50,70, and $90 \%$ of the peak flux (from left to right for $\mu=2$ the continuum peak flux is $0.43,0.26$, and $0.23 \mathrm{Jy} \mathrm{beam}^{-1}$; for $\mu=17$ is it $0.62,0.74$, and $0.72 \mathrm{Jy}_{\text {beam }}^{-1}$ ). ALMA would reach the required $0.1 \%$ linear polarization sensitivity in approximately $5 \mathrm{~min}$ on source integration time

\section{Conclusion}

ALMA will provide a several orders of magnitude improvement in polarimetric capabilities at the (sub-)millimetre wavelengths compared to the currently available instruments such as the SMA. Not only will the enhanced sensitivity allow for the imaging of magnetic fields in much larger samples of sources, but it will also allow for magnetic field imaging of dense and dusty regions in galaxies besides our own. By imaging the linear polarization of a variety of spectral lines, probing different regions in circumstellar and protostellar envelopes and outflows, it will be possible to generate a detailed picture of the magnetic field morphology. Applying the Chandrasekhar-Fermi method would then allow for measurements of the projected magnetic field strength at various densities. Added to this, circular polarization observations will be 
possible for a small number of molecules, such as $\mathrm{CN}$, and will provide the line-of-sight magnetic field. Furthermore, ALMA polarization will also be able to constrain, for example, maser pumping models and models for the supermassive black hole Sgr A* at the Galactic Center.

The rapid increase in polarization observations that ALMA will produce will also likely provide challenges to several current theoretical models on the generation of polarized dust and line emission itself. Several of these theoretical descriptions are detailed in other chapters of this book, but they include dust alignment as well as the study of dust composition.

Finally, ALMA will be able to produce polarization data products even when this is not the main observational goal. Combined with the new developed analysis tools, such as those from the ARTIST project, the observations of magnetic fields will become increasingly accessible. Thus, ALMA polarization capabilities will usher in an exciting new era in the study of magnetic fields at (sub-)millimeter wavelength.

Acknowledgements WV would like to thank Marco Padovani and Susanne Aalto for providing figures and Daniel Tafoya and Kirsten Kraiberg Knudsen for feedback on the manuscript.

\section{References}

Aalto, S., Hüttemeister, S.: Astron. Astrophys. 362, 42 (2000)

Allen, A., Shu, F.H., Li, Z.-Y.: Astrophys. J. 599, 351 (2003)

Amiri, N., Vlemmings, W.H.T., Kemball, A.J., van Langevelde, H.J.: Astron. Astrophys. 538, A136 (2012)

Balick, B., Frank, A.: Ann. Rev.Astron. Astrophys. 40, 439 (2002)

Bartkiewicz, A., Szymczak, M., Cohen, R.J., Richards, A.M.S.: Mon. Not. R. Astron. Soc. 361, 623 (2005)

Beck, R., Fletcher, A., Shukurov, A., et al.: Astron. Astrophys. 444, 739 (2005)

Beuther, H., Vlemmings, W.H.T., Rao, R., van der Tak, F.F.S.: Astrophys. J. Lett. 724, L113 (2010)

Brinch, C., Hogerheijde, M.R.: Astron. Astrophys. 523, A25 (2010)

Bujarrabal, V., Castro-Carrizo, A., Alcolea, J., Sánchez Contreras, C. Astron. Astrophys. 377, 868 (2001)

Castro-Carrizo, A., Quintana-Lacaci, G., Neri, R., et al.: Astron. Astrophys. 523, A59 (2010)

Chandrasekhar, S., Fermi, E.: Astrophys. J. 118, 116 (1953)

Cortes, P.C., Crutcher, R.M., Watson, W.D.: Astrophys. J. 628, 780 (2005)

Cotton, W.D.: MMA Memo 208 (1998)

Crutcher, R.M., Troland, T.H., Kazes, I.: Astron. Astrophys. 181, 119 (1987)

Crutcher, R.M., Roberts, D.A., Mehringer, D.M., Troland, T.H.: Astrophys. J. Lett. 462, L79 (1996)

Escoffier, R. P., Comoretto, G., Webber, J.C., et al.: Astron. Astrophys. 462, 801 (2007)

Falceta-Gonçalves, D., Vidotto, A.A., Jatenco-Pereira, V.: Mon. Not. R. Astron. Soc. 368, 1145 (2006)

Falgarone, E., Troland, T.H., Crutcher, R.M., Paubert, G.: Astron. Astrophys. 487, 247 (2008)

Fiebig, D., Guesten, R.: Astron. Astrophys. 214, 333 (1989)

Fish, V.L., Reid, M.J.: Astrophys. J. Suppl. 164, 99 (2006) 
Frank, A., De Marco, O., Blackman, E., Balick, B.: arXiv:0712.2004 (2007)

Frau, P., Galli, D., Girart, J.M.: Astron. Astrophys. 535, A44 (2011)

Fromang, S., Hennebelle, P., Teyssier, R.: Astron. Astrophys. 457, 371 (2006)

Gaensler, B.M., Haverkorn, M., Staveley-Smith, L., et al.: Science 307, 1610 (2005)

Girart, J.M., Crutcher, R.M., Rao, R.: Astrophys. J. Lett. 525, L109 (1999)

Girart, J.M., Rao, R., Marrone, D.P.: Science 313, 812 (2006)

Girart, J.M., Beltrán, M.T., Zhang, Q., Rao, R., Estalella, R.: Science 324, 1408 (2009)

Girart, J.M., Patel, N., Vlemmings, W., Rao, R.: Astrophys. J. 751, L20 (2012)

Glenn, J., Walker, C.K., Bieging, J.H., Jewell, P.R.: Astrophys. J. Lett. 487, L89 (1997)

Goldreich, P., Kylafis, N.D.: Astrophys. J. Lett. 243, L75 (1981)

Goldreich, P., Kylafis, N.D.: Astrophys. J. 253, 606 (1982)

Gómez, Y., Tafoya, D., Anglada, G., et al.: Astrophys. J. 695, 930 (2009)

Greaves, J.S., Holland, W.S., Minchin, N.R., Murray, A.G., Stevens, J.A.: Astron. Astrophys. 344, 668 (1999)

Greaves, J.S., Holland, W.S., Jenness, T., Hawarden, T.G.: Nature 404, 732 (2000)

Herpin, F., Baudry, A., Thum, C., Morris, D., Wiesemeyer, H.: Astron. Astrophys. 450, 667 (2006)

Herpin, F., Baudy, A., Josselin, E., Thum, C., Wiesemeyer, H.: IAU Symp. 259, 47 (2009)

Kemball, A.J., Diamond, P.J.: Astrophys. J. Lett. 481, L111 (1997)

Krause, F., Beck, R.: Astron. Astrophys. 335, 789 (1998)

Kuiper, R., et al.: In prep.

Kwok, S., Purton, C.R., Fitzgerald, P.M.: Astrophys. J. Lett. 219, L125 (1978)

Lazarian, A.: J. Quant. Spectrosc. Radiative Transf. 79, 881 (2003)

Leone, F., Martínez González, M.J., Corradi, R.L.M., Privitera, G., Manso Sainz, R.: Astrophys. J. Lett. 731, L33 (2011)

Li, H.-B., Henning, T.: Nature 479, 499 (2011)

Mestel, L., Spitzer, L. Jr.: Mon. Not. R. Astron. Soc. 116, 503 (1956)

Morris, M., Lucas, R., Omont, A.: Astron. Astrophys. 142, 107 (1985)

Ostriker, E.C., Stone, J.M., Gammie, C.F.: Astrophys. J. 546, 980 (2001)

Padovani, M., Brinch, C., Girart, J.M., et al.: Astron. Astrophysics. 543, 16 (2012)

Padovani, M., et al.: IAU Sump. 270, 451 (2011)

Robishaw, T., Quataert, E., Heiles, C.: Astrophys. J. 680, 981 (2008)

Sabin, L., Zijlstra, A.A., Greaves, J.S.: Mon. Not. R. Astron. Soc. 376, 378 (2007)

Sarma, A.P., Troland, T.H., Romney, J.D.: Astrophys. J. Lett. 554, L217 (2001)

Surcis, G., Vlemmings, W.H.T., Dodson, R., van Langevelde, H.J.: Astron. Astrophys. 506, 757 (2009)

Surcis, G., Vlemmings, W.H.T., van Langevelde, H.J., Hutawarakorn Kramer, B.: Astron. Astrophys. 541, A47 (2012)

Szymczak, M., Cohen, R.J., Richards, A.M.S.: Mon. Not. R. Astron. Soc. 297, 1151 (1998)

Tang, Y.-W., Ho, P.T.P., Koch, P.M., et al.: Astrophys. J. 700, 251 (2009)

Teyssier, R.: Astron. Astrophys. 385, 337 (2002)

Thum, C., Morris, D.: Astron. Astrophys. 344, 923 (1999)

Vlemmings, W.H.T.: Astron. Astrophys. 484, 773 (2008)

Vlemmings, W.H.T., van Langevelde, H.J., Diamond, P.J.: Astron. Astrophys. 434, 1029 (2005)

Vlemmings, W.H.T., Diamond, P.J., Imai, H.: Nature, 440, 58 (2006)

Vlemmings, W.H.T., Diamond, P.J., van Langevelde, H.J., Torrelles, J.M.: Astron. Astrophys. 448, 597 (2006)

Vlemmings, W.H.T., Surcis, G., Torstensson, K.J.E., van Langevelde, H.J.: Mon. Not. R. Astron. Soc. 404, 134 (2010)

Vlemmings, W.H.T., Humphreys, E.M.L., Franco-Hernández, R.: Astrophys. J. 728, 149 (2011)

Vlemmings, W.H.T., Ramstedt, S., Rao, R., Maercker, M.: Astron. Astrophys. 540, L3 (2012)

Wolak, P., Szymczak, M., Gérard, E.: Astron. Astrophys. 537, A5 (2012) 